Title: Surprising sequential effects on MMN

\title{
Authors:
}

Frost, J. D ${ }^{1}$

Winkler, I. ${ }^{2}$

Provost, A. ${ }^{1}$

Todd, J.

1. School of Psychology, University of Newcastle, Australia.

2. Research Centre for Natural Sciences, MTA, Institute of Cognitive Neuroscience and Psychology, Budapest, Hungary

Corresponding author:

Juanita Todd

School of Psychology, University of Newcastle

University Drive, Callaghan, NSW, Australia, 2308.

Email: Juanita.Todd@newcastle.edu.au

Phone: +612 49215977

Fax: +6124921 


\begin{abstract}
The mismatch negativity (MMN) is conceptualised as a confidence-weighted error signal elicited when a deviation violates the predicted next-state based on regularity. The mechanisms underpinning its generation remain contentious. Smaller MMN response is a robust finding in schizophrenia and reduced amplitude may implicate impairment in prediction-error signalling. An enriched understanding of factors that influence MMN size in healthy people is a prerequisite for translating the relevance of reduced MMN in schizophrenia. This paper features two studies designed to explore factors that impact MMN in healthy individuals. Study 1 confirms that MMN amplitude does not faithfully reflect transition statistics and is susceptible to order-driven bias. In Study 2, we demonstrate that an order-driven bias remains despite repeated encounters with sound sequences. These data demonstrate that factors that impact on MMN size in non-clinical groups are not fully understood and that some mechanisms driving relevance filtering are likely influenced by ‘top-down' expectations.
\end{abstract}

Keywords: Schizophrenia, context, bias, first-impressions, ERP, Auditory, Mismatch Negativity.

Highlights

- The mechanisms that underpin MMN generation remain a contentious issue.

- Understanding MMN in healthy people is a prerequisite for clinical applications.

- We confirm order-driven bias modulates MMN amplitude in role-alternating sequences.

- We show that this bias remains despite repeated encounters with sound sequences.

- Relevance filtering is likely influenced by ‘top-down' expectations. 


\section{Surprising sequential effects on MMN}

Research on the mismatch negativity (MMN) component of the auditory event-related potential (ERP) has flourished over the last 35 years with peer-reviewed publications on this topic exceeding 2000 and more than 200 of these have explored its characteristics in persons diagnosed with schizophrenia. The robust observation that MMN amplitude is reduced in schizophrenia has spawned a prolific effort to validate its utility as a biomarker in this group (see Atkinson, Michie, \& Schall, 2012; Bodatsch et al., 2011; Stephan, Baldeweg, \& Friston, 2006; Gil-da-Costa, Stoner, Fung, \& Albright, 2013; Javitt, Zukin, Heresco-Levy, \& Umbricht, 2012; Light et al., 2012). In both persons with schizophrenia and healthy comparison groups the MMN is elicited following the presentation of a rare, unexpected sound within a repeating stimulus pattern (Javitt et al., 1998). Yet, the signal is typically significantly smaller schizophrenia than it is in healthy controls. Evidence suggests MMN is elicited only when a prediction model containing information about regularity exists and that the brain uses this model to anticipate future sensory input and minimise prediction-error (Winkler et al., 1996; Winkler 2007; Winkler \& Czigler, 2012). It is therefore possible that smaller MMN in those with schizophrenia indexes impairment in predictive processing (Stephan et al., 2006; Todd et al., 2012). However, a necessary prerequisite for interpreting reduced MMN in those with schizophrenia is the need for a comprehensive understanding of the factors that impact MMN amplitude more generally. It is this pursuit that has uncovered highly unexpected order-driven influences on MMN in a "multi-timescale" paradigm (Todd, Provost, \& Cooper, 2011). This bias contradicts the notion that MMN will always be larger in amplitude when a pattern has been stable for longer and exploring the cases and limits of this bias can help us better understand the timescales of memory influencing MMN amplitude.

This paper features two studies designed to explore why MMN amplitude in healthy populations doesn't always increase uniformly when patterns are more stable. In study 1 we report a replication of the patterns of order-driven bias in MMN amplitude in a much larger dataset and in 
study 2 we demonstrate the persistence of this bias despite repeated exposure to sound sequences that should theoretically abolish its affect. Finally we discuss how these order effects may help provide a deeper understanding of factors impacting MMN amplitude in non-clinical groups and emphasize there is still much to be learned for MMN that may influence its use as a meaningful tool in clinical groups such as schizophrenia.

\section{MMN and the Multi-timescale Design}

In a classic oddball paradigm, MMN will be observed in the auditory ERP when a sound violates regularity within a repetitious sequence (Näätänen, 1992). The sound may contain a rare physical feature deviation (as in most schizophrenia studies), a rare combination of features or may occur with unexpected timing (e.g., unexpectedly early or late repeat; Kujala, Tervaniemi \& Schröger, 2007). Increasingly, prominent views conceptualize the brain as capable of sophisticated hypothesis-testing and of using internal models to make predictions about future states of the sensory world (Winkler, 2007; Winkler \& Schröger, 2015; Friston, 2005; Friston \& Stephan, 2007). From this perspective, the MMN is considered a prediction-error signal that indicates the degree to which an expected state differs from that which is actually experienced. As models of the processes underling MMN have matured, it is clear that MMN amplitude is tightly coupled to some quantification of "confidence" in the underlying predictions about future sound properties (Lieder, Daunizeau, Garrido, \& Friston, 2013; Winkler, 2007). Expressed simply, the confidence in an active prediction model will depend on the variability of incoming sensory input. A large amount of variation is apparent when the environment is highly unstable and this undermines confidence that the active model can accurately predict incoming stimuli. Predictions associated with high confidence are observed when regularity is extremely simple (e.g., a repeating identical tone) and highly stable/probable (Lieder et al., 2013). Confidence accumulates each time predictions successfully match sound input and this leads to a more accurate model with high precision. A possible ERP correlate of trace/prediction accumulation is the so-called repetition positivity (RP; 
Haenschel et al., 2005. Deviations from predictions that occur under conditions of high confidence will elicit a significantly larger MMN error signal (manifest as increased negativity in evoked potentials in fronto-central scalp electrodes 80-250 ms after detected deviation).

Most studies investigating MMN in schizophrenia use relatively simple sound sequences (e.g. sounds deviating on no more than a few features; see Todd, Harms, Schall, \& Michie, 2012 for review). However the exploration of MMN in alternate paradigms continues to facilitate a deeper understanding of normal predictive processes because the MMN responses elicited in stimulus paradigms with dynamically changing standard/deviant configurations reveal how the auditory system adjusts its predictions in accordance with the changing roles of the sounds (Cowan, Winkler, teder, \& Näätänen et al., 1993; Garrido et al., 2008; Winkler, Karmos, \& Näätänen, 1996). Studies varying standard/deviant probabilities within subjects have shown that MMN amplitude plateaus at a certain point in controls and it plateaus at lower amplitude in patients with schizophrenia (Sato et al., 2003; Shelley, Silipo, \& Javitt, 1999; Javitt, Grochowski, Shelley, \& Ritter, 1998) while Baldeweg and colleagues (2004) have found that the number of standard-stimulus repetitions needed to establish confident predictions correlated with the severity of schizophrenic symptoms. It is therefore possible that the predictive processing system is less than optimal in persons with schizophrenia (Todd et al., 2012). One way in which impaired processing could arise is through a limitation in the period over which confidence in a prediction model accumulates. For example, a system that can store accumulated confidence in a model over a period encapsulating 100 events could adjust response characteristics to differentially code errors as rare as $1 \%$ while a system limited to 20 events could not distinguish between an error frequency of 5\% and $1 \%$ (such as in controls versus patients, see Figure 2 in Javitt et al., 1998).

The design principle in the multi-timescale paradigm was to keep local standard/deviant probabilities constant ( 0.875 and 0.125 , respectively) and explore how changes in the longer-term stability of these patterns affected MMN in healthy people(Todd et al., 2011). The sequences within the multi-timescale paradigm contain two physically different sounds that alternate standard 
and deviant roles over time (see Figure 1). Previous research shows that perceptual inferences reflected in prediction models are updated very dynamically such that a model will be altered after as few as 2-3 consecutive errors causing a new repetition (Bendixen, Prinz, Horvath, TrujilloBarreto, \& Schroger, 2008; Sams, Alho, \& Näätänen, 1983). The properties of a former deviant will be incorporated into a new prediction model after a few repetitions, so a former standard in the multi-timescale sequence will come to elicit MMN when it occurs as a rare deviation from this new context. In separate sequences within the multi-timescale paradigm, the period of standard/deviant stability is varied such that in fast changing "unstable" sequences, the roles reverse after 160 tones (0.8 mins) and in "stable" sequences, they reverse after 480 tones (2.4 minutes). The design was based on the expectation that participants affected by a long history of sound would show larger MMN in stable than unstable sequences. However if participants were only affected by a short history, for example $<0.8$ minutes, this should result in minimal differences in MMN amplitude in the different stability sequences. The surprise finding was that both patterns were in fact present in the same participants. MMN to deviant sounds in those periods in which the standard/deviant configuration matched the one encountered at the sequence onset (first-deviants) showed a clear stable > unstable result, while MMN to deviant sounds encountered in periods in which the roles had been reversed (second-deviants) was equivalent in amplitude for both sequences (Todd et al., 2011).

In Todd et al., (2011), role stability also had an impact on ERPs to standard sounds which emerged in the ERP as an apparent increase in positivity (although technically the combined effect of decreased N1 and increased P2) in stable relative to unstable sequences. However, this effect was consistent across tone type (i.e., no order effect) leading the authors to deduce that the bias phenomenon is driven by changes in ERPs to the deviant sound and does not modulate the RP appearing in the standard-stimulus response. In subsequent studies, the same basic pattern of bias has been observed to different feature deviations and so is clearly tied to the first-deviant, second- 
deviant status of the tones (Todd, Provost, Whitson, Cooper, \& Heathcote, 2013a; Todd et al., 2013b; Todd et al., 2014; Mullens et al., 2014).

A similar order-driven effect on MMN amplitude has been observed (Costa-Faidella, Grimm, Slabu, Díaz-Santaella \& Escera, 2011) in a study designed to replicate animal work showing long timescale stimulus specific adaptation effects on standard and deviant ERPs (Ulanovsky, Las, Farkas \& Nelken, 2004). Stimulus-specific adaptation (SSA) refers to the reduced spiking rate in neural response to a repeated stimulus. The paradigm used by Costa-Faidella et al. featured much shorter sequences $(25 \mathrm{sec})$ containing two tones that switch roles as standard and deviant midway through. The authors observed that MMN amplitude to first standard when it later occurred as a deviant (after the switch) was smaller overall due to an initial "suppression" of response immediately after the roles reversed. This suppression of response to a deviant with a long prior history of repetition was attributed to the possible existence of a subgroup of auditory neurons that exhibit adaptation to sound probability on multiple timescales.

The multi-timescale paradigm revealed that MMN size following a pattern violation is susceptible to order-driven bias and is not a simple function of pattern stability and local sound statistics. An important consideration in understanding this phenomenon is whether this can be accounted for by factors sometimes referred to as "bottom-up" (meaning a modulation of response that can be accounted for by the history of sensory input) or "top-down" (meaning a modulation of response that is imposed on the auditory system by an explicit prediction-model). The degree to which bottom-up and top-down factors account for MMN has attracted considerable debate (see Näätänen, Jacobsen \& Winkler, 2005 for discussion). Current views suggest that both can contribute to the generation of this ERP component (Sussman, 2007) and that the bottom-up contribution is required (Sadia, Ritter \& Sussman, 2013). Indeed the degree to which each contribute to smaller MMN in schizophrenia has also attracted debate (Baldeweg, Klugman, Gruzelier \& Hirsch, 2006, Dima, et a., 2012). An order-driven impact on MMN amplitude could conceivably be reliant on either top-down or bottom-up effects on the ERP. The order-driven MMN 
modulation observed by Costa-Faidella and colleagues (2011) probably exemplifies a bottom-up effect because it mirrors the SSA finding of Ulanovsky and colleagues (2004), which is generally accepted to be a "bottom-up" contribution to the process generating MMN.

Although the order effects in the multi-timescale sequences occur over much longer timeframes, it is likely that similar mechanisms contribute to the observed bias. However, at least two observations indicate that top-down effects must also contribute to MMN patterns in this data. Firstly, the effect of tone order on MMN amplitude in multi-timescale sequences is actually sensitive to the behavioural relevance of the sounds. In Mullens et al (2014) participants initially heard the two sounds with equal probability and were asked to respond as quickly and accurately as possible to either the shorter sound (short-go group) or the longer sound (long-go group). The appearance of order-effects on MMN amplitude in a subsequent multi-timescale sequence differed in these two groups in a task-dependent manner. This result cannot be explained by "bottom-up" influences because the two groups heard the sounds with exactly the same probabilities and orders; the only difference was what they were asked to do in response to the sounds.

The second observation indicating that the order-effects observed in the multi-timescale sequences may reflect top-down influence is the focus of study 1 in the present paper. It was previously observed that the order effect on MMN amplitude is different in slowly changing (stable) sequences than in subsequent faster-changing (unstable) sequences in the multi-timescale paradigm. Explaining this differential pattern of data imposes a strong assumption on the bottom-up adaptation based explanation of the order-driven effects: The strong adaptation developed for the standard in the final block of the stable sequence suppresses the response to this tone when encountered as the deviant in the following unstable sequence. If this was the case, the adaptation-based bias should diminish during the course of the alternations of the unstable sequence. Testing this possibility requires averaging low number of trials per participant and thus requires a larger group for sufficient statistical power. In study 1, we determine whether the finding of different effects in stable vs. unstable sequences can be replicated in an expanded dataset and to test whether the 
differential effect is confined to the initial segment of the unstable sequence. In study 2, participants were presented with four occurrences of either the stable sequence or the unstable sequence to determine whether the order-effects would diminish with repeated exposure. We ask whether assuming the presence of top-down influences is necessary to account for the results of both studies. Implications about general factors impacting MMN amplitude are also discussed.

\section{Method - Study 1}

\section{Participants.}

The data comprised 14 individual data sets from Todd et al. (2013b) plus an additional 21 unpublished data sets collected subsequently under identical conditions. The data therefore consisted of 35 (22 females, $18-33$ years, mean $=23$ years, $\mathrm{SD}=4$ years $)$ healthy community volunteers and undergraduate students from the University of Newcastle, Australia. All data sets were collected under standards approved by the local Human Research Ethics Committee. A structured interview was conducted by one of the researchers to ensure all participants met criteria for inclusion which stipulated normal hearing, no history of head injury of neurological condition, no current mental illness or family history of psychosis and no alcohol or substance abuse. Remuneration was offered as course credit to students and cash reimbursement to community volunteers. Written informed consent was obtained from all participants consistent with standards approved by the Human Research Ethics Committee.

\section{Stimuli and sequences.}

Sounds were $1 \mathrm{kHz}$ pure tones presented binaurally over headphones at $75 \mathrm{~dB}$ SPL with 5 $\mathrm{ms}$ rise/fall times and either a $20 \mathrm{~ms}$ or a $50 \mathrm{~ms}$ pedestal to produce $30 \mathrm{~ms}$ and $60 \mathrm{~ms}$ sounds, respectively. Sounds within sequences were arranged within long-deviant and short-deviant blocks. In long-deviant blocks, $30 \mathrm{~ms}$ tones were highly probable $(p=0.875)$ and $60 \mathrm{~ms}$ tones were rare deviants $(p=0.125)$; in short-deviant blocks, the tone probabilities were reversed (30 $\mathrm{ms}$ and $60 \mathrm{~ms}$ presented at $p=0.875$ and 0.125 , respectively; see Figure 1). In separate sequences, long- and 
short-deviant blocks were presented at either slow or fast block-alternation speeds (hereafter referred to as stable and unstable sequences, respectively) with sequences always commencing with long- preceding short-deviant (hereafter referred to as first and second-deviant, respectively). Each sequence consisted of 1920 tones in total, 960 of each duration. Tones were presented at a regular $300 \mathrm{~ms}$ stimulus onset asynchrony (9.6 min per sequence). In the stable sequences, sounds were arranged in four blocks with block-type type alternating after every 480 sounds, producing a stablestandard period of $2.4 \mathrm{~min}$. In the unstable sequence, sounds were arranged in twelve blocks with block-type alternating every 160 tones, creating a stable-standard period of 0.8 min. Note, full counterbalancing of sequence order and tone role as first or second-deviant was not performed here but was present in Todd et al. 2011 and counterbalancing of first-deviant identity was also present in Mullens et al. 2014 and Todd et al 2014.
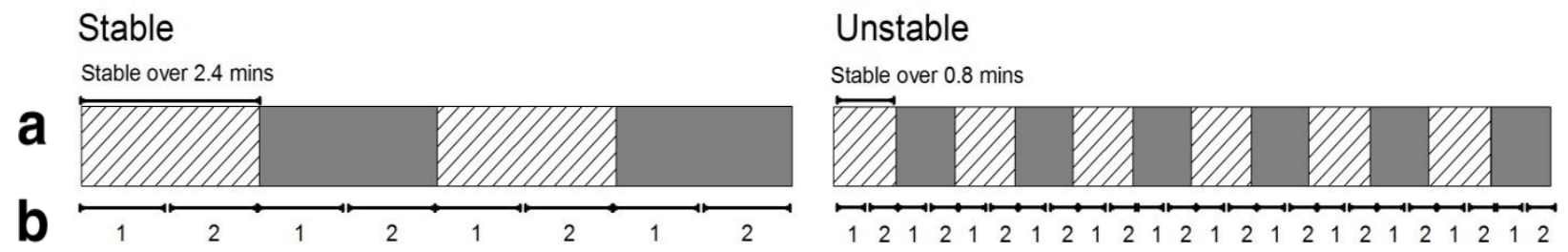

Figure 1. A diagram illustrating the stable and unstable sound sequences (Todd et al., 2013a) a The diagonal lined rectangles represent one deviant block type and the greyed rectangles, the other. In the blocks marked with diagonal lines, the long $(60 \mathrm{~ms})$ sound was the rare $(p=0.125)$ deviant and the short $(30 \mathrm{~ms})$ sound was the highly probable $(p=.875)$ standard. In the greyed blocks, the probabilities were reversed. The block length within stable and unstable sequences were 2.4 and 0.8 mins respectively, creating a difference of stability in the relative tone probabilities $\mathbf{b}$ Sections labelled 1(first-half data) and 2 (second-half data) show how blocks were divided for halvesanalysis as detailed in Todd et al. (2013a). Averages for MMN to the deviants were created for both stable and unstable conditions by pooling together the responses for first-halves (i.e. the point in which tone roles transition) to produce first-half long- and short-deviant MMNs. Averages for MMN to the deviants over the latter portion of the blocks for each condition were generated by 
averaging together the responses for second-halves to create second-half long- and short-deviant MMNs.

\section{Procedure.}

All participants completed a screening interview to ensure inclusion criteria were met. An audiometric screen using a pure tone audiometer (Earscan ES3S) across $500 \mathrm{~Hz}-4000 \mathrm{~Hz}$ was used to assess hearing thresholds $(\leq 25 \mathrm{~dB}$ SPL $)$ to ensure absence of hearing loss. Participants were then fitted with a Neuroscan Quikcap with $\mathrm{Ag} / \mathrm{AgCl}$ electrodes. The continuous EEG was recorded on a Synamps 2 Neuroscan system at $1000 \mathrm{~Hz}$ sampling rate (highpass $0.1 \mathrm{~Hz}$, lowpass $70 \mathrm{~Hz}$, notch filter $50 \mathrm{~Hz}$ and a fixed gain of 2010). EEG data were collected from 13 electrode locations (FZ, CZ, PZ, F3, FC3, C3, F4, FC4, C4, F7, F8 in accordance with the 10-20 system, plus left and right mastoid) and referenced to the nose. In addition, vertical and horizontal electro-oculograms were recorded using electrodes placed above and below the left eye, and $1 \mathrm{~cm}$ from the outermost canthus of each eye to monitor eye-blinks and -movements. All impedances were reduced to below $5 \mathrm{k} \Omega$. Sequences were presented over headphones (Sennheiser HD280pro) while the participant viewed a film (sound muted) with sub-titles. Participants were told they would hear sounds over the headphones but that the brain responses we were studying were automatic and best recorded if they could try to ignore the sounds and focus attention on the film.

\section{Data Analysis.}

The continuous EEG recording was examined offline for major artefact and corrected for eye blinks using the procedures in Neuroscan Edit Software. This method applies a regression analysis in combination with artefact averaging (Semlitsch, Anderer, Schuster, \& Presslich, 1986). The values generated was assessed for adequacy ( $>30$ sweeps in the average and $<5 \%$ variance) and applied to the continuous data file. Each file was epoched from $50 \mathrm{~ms}$ pre-stimulus to $300 \mathrm{~ms}$ post-stimulus. Epochs were baseline corrected to the pre-stimulus interval and averaged according to stimulus type. Epochs containing variations exceeding $\pm 70 \mu \mathrm{V}$ were excluded. 
Standard and deviant ERPs were created separately for the period equating to the first-half of blocks (0-1.2 minutes for blocks in stable sequences and 0-0.4 minutes for blocks in unstable sequences) and for second-half of blocks (1.2-2.4 minutes for blocks in stable sequences and 0.4-0.8 minutes for blocks in unstable sequences; see Figure 1). This was essential as Todd et al. (2013a) observed the bias effects to be prominent in first-half data only. Data for stable and unstable sequences were used to create four ERPs to standard tones and four to deviant tones (first-halffirstdeviant, second-half first-deviant, first-half second-deviant, second-half second-deviant). All standard and deviant ERPs were digitally filtered with a $30 \mathrm{~Hz}$ lowpass filter. Difference waveforms were generated by subtracting the ERP to a sound as a standard in the first- or secondhalf of blocks from the ERP to that same sound as a deviant in the same block period for each sequence. The majority of participants had between 50 and 60 deviant sweeps contributing to averages with a minimum of 43 for any deviant waveforms. MMN amplitude was quantified by extracting the mean peak amplitude over a $20 \mathrm{~ms}$ period centred on the most negative point 100-250 ms post-stimulus onset. As per Todd et al. (2013a), a total of eight MMN values were compared at F4 (where the MMN was maximal) in repeated measures ANOVA with sequence (stable, unstable), deviant (first-deviant, second-deviant) and half (first, second) as within-subject factors. Paired (twotailed) t-tests were performed for a-priori simple effects with p-value set at .05 .

\section{Results.}

Difference waveforms for the first and second-deviant in stable and unstable sequences across first and second block halves are presented in Figure 2 and the corresponding mean amplitude values are presented in Figure 3. There is a clear MMN evident for each of the sequence types, for each of the deviants and for both the first and second half of sequence blocks. However, in the stable sequence (Figure 2A), MMN appears to increase more over the first to second half of blocks for the second-deviant than the first, while the reverse appears to be true for the unstable sequence (Figure 2B). Analysis revealed main effects of sequence (stable generally larger than unstable, $F(1,34)=14.84, p<.001, \eta 2=.30$ ) and half (second-half generally larger than first-half, 
$F(1,34)=10.64, p<.01, \eta 2=.24)$ but these patterns were further modified by a significant deviant $\mathrm{x}$ sequence $\mathrm{x}$ half $(F(1,34)=7.77, p<.01, \eta 2=.19)$ interaction meaning that the stability effects reflected in sequence and half were different for the first-deviant and second-deviant MMNs.

A. Stable Sequence

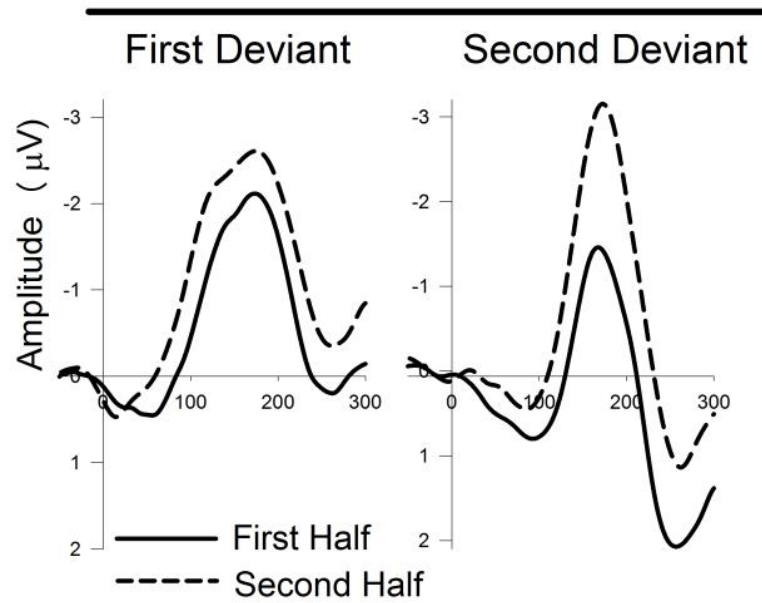

B. Unstable Sequence

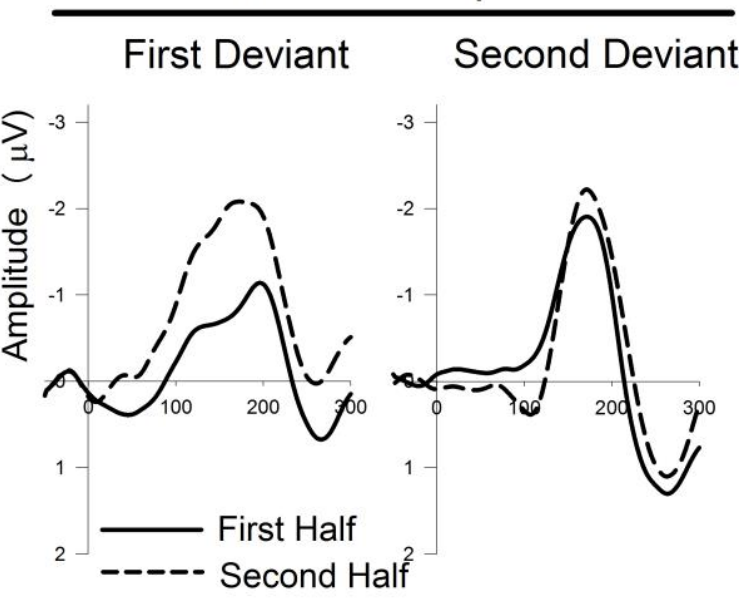

Figure 2. Difference waveforms in A. Stable Sequence and B. Unstable Sequence for first (60 ms) and second (30 ms) deviant. Difference waveforms for data obtained in the first-half of blocks (solid lines) are presented with those in the second-half of blocks (broken lines).

For the first-deviant there was a main effect of sequence (stable $>$ unstable, $F(1,34)=15.39$, $\mathrm{p}<.001, \eta 2=.31)$ and half (second $>$ first, $\mathrm{F}(1,34)=6.02, \mathrm{p}<.05, \eta 2=.15$ ) with no interaction. However, planned analyses revealed that the MMN only increased significantly over halves for the unstable sequence $(t(34)=2.77, p<.01)$ and the effect of sequence was significant in first-half data only $(t(34)=4.04, p<.001)$ and this is clear in Figure 3A. In contrast, for the second-deviant there were no main effects but a significant sequence by half interaction $(F(1,34)=8.36, \mathrm{p}<.01, \eta 2=$ .20). This was due to MMN increasing significantly over halves for the stable sequence only (see Figure $3 \mathrm{~B}, t(34)=3.01, p<.005)$ and the effect of sequence only being significant for the second half of blocks $(t(34)=2.93, p<.01)$. 
First Deviant

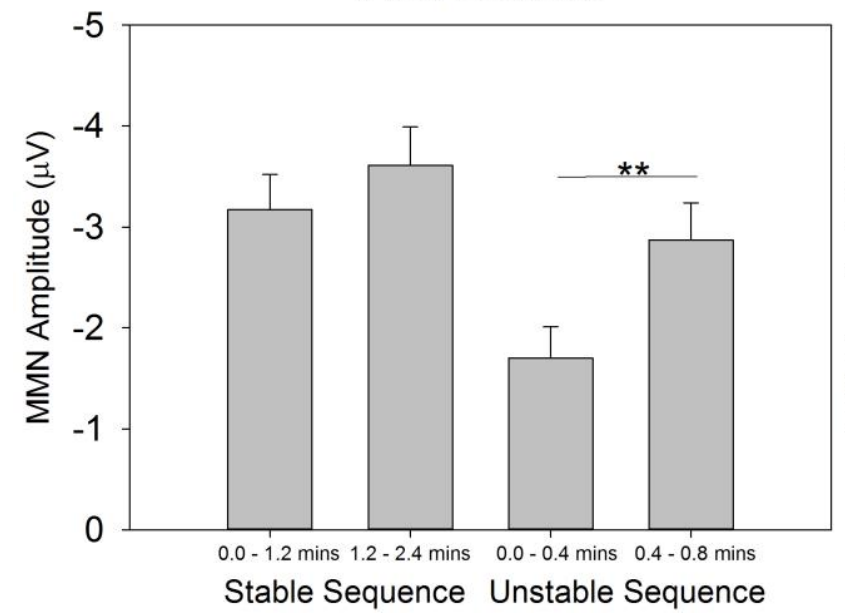

Second Deviant

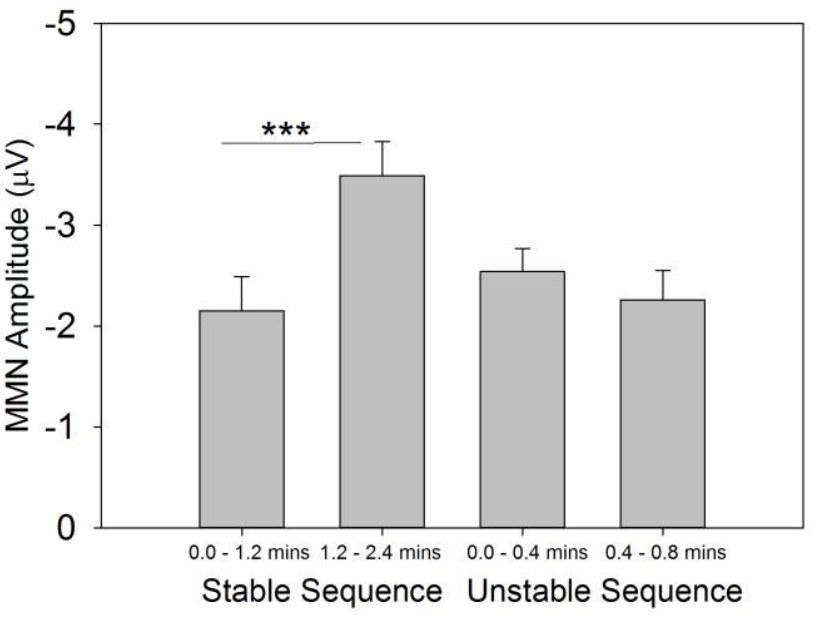

Figure 3. Group mean amplitude for mismatch negativity (MMN) for the first-deviant (left) and second-deviant (right) in first and second halves of stable and unstable sequences. Asterisks denote significant difference across a sequence block type. $* *=p<0.01, * * *=p<0.001$.

The reverse pattern of block half effects in the Stable and Unstable sequences could potentially be explained by the long period of $60 \mathrm{~ms}$ standard tones at the end of the Stable sequence leading to reduced response to these tones as deviants in the subsequent Unstable sequence. This could be expected to result in differential changes in Unstable sequence MMNs for the first and second deviants over early versus later blocks of the sequence. Early MMN (to deviants in the first 3 blocks) versus later MMN (to deviants in the second 3 blocks) was compared over tones in repeated measures ANOVA. The analysis yielded no significant main effects nor interactions. Means for early and late $\mathrm{MMN}$ for the first-deviant $(\mathrm{m}=-2.03, \mathrm{sd}=2.31$ and $\mathrm{m}=-2.08, \mathrm{sd}=2.08$, respectively) and for the second-deviant $(\mathrm{m}=-2.54, \mathrm{sd}=1.79$ and $\mathrm{m}=-1.77, \mathrm{sd}=2.07$, respectively) were very similar but the evidence was not strong enough to support early and later block values coming from a sample with the same mean based on a city-block distance test (Widmann \& Schröger, 1999). 


\section{Discussion.}

The larger data set presented here $(n=35)$ replicates the finding that MMN amplitude is differently affected in the multi-timescale sequences for the first and second-deviant tones (Todd et al., 2013b). When examining stability effects within the stable slowly changing sequence MMN amplitude for the first-deviant is already large in first-half data and stays large over second-half data showing minimal growth from the period when roles were initially established versus later in the block when confidence has had additional time to accumulate. In contrast, MMN to the seconddeviant tone is significantly smaller in first-half of the blocks than in the second-half of blocks showing a clear stability effect. This observation is consistent with that reported in Todd et al., 2013 and is also consistent with that of Costa-Faidella et al., (2011) where MMN to a deviant with a long prior history as a standard is initially very small but recovers with increasing stability in the new pattern. As per Costa-Faidella, no order-patterns were reflected in the standard ERPs, but only in deviant ERPs and resultant MMNs (see Supplementary analysis).

The data obtained in the unstable sequence exhibits the opposite pattern even though the sequences commence with the same standard and deviant roles. For unstable sequences, MMN to the first-deviant is initially small but increases significantly across block halves, while that to the second deviant is actually as large during the first-half of the blocks as it is by the second-half. In fact, it is evident from Figure 3B that the MMN obtained after a period of 0.4 minutes stability in the unstable sequence data is as large as that obtained after 1.2 minutes of stability in the first-half of the stable sequence blocks.

Can long timescale adaptation of afferent neuronal assemblies explain the reversal of ordereffects on MMN in the unstable following stable sequence? One possible explanation could relate to the first-deviant being the highly repetitious standard in the final block of the stable sequence. If a strong adaptation is developed it could potentially influence the response to this tone as a deviant in the following unstable sequence. If this were the case one might expect this effect to diminish over the course of the sequence leading to larger amplitude MMNs to the first-deviant in later blocks. 
There was no evidence in the data that this was the case so while we cannot rule out an adaptation explanation the data do not provide strong support for it. Incidentally the absence of a significant difference in MMN for the early and late blocks of the Unstable sequence occurs in the presence of a significant difference between the first and second halves of these blocks. Although it is difficult to draw conclusions from these subgroupings of data it is certainly consistent with Todd et al.'s (2013) suggestion that the difference over block halves is something that is repeated each time the blocks are encountered.

Todd et al., 2013 alternatively proposed that the order-effects may be explained by a lasting "first-impression". They hypothesized that the standard and deviant roles (or probabilities) first encountered at sequence onset are "locked in" as a type of prior - that is, an assumption about the probability distribution defining the likelihood and/or importance of future events (Griffiths et al. 2008). The repetitive standard tone is rapidly established to be a predictable event providing minimal new information while the deviant occurs unexpectedly as a potentially relevant violation of expectations. When the roles are encountered in the reverse order, there is initially very low confidence that the former repetitive standard is now an informative error signal. It is recognised as an error relative to the new prediction model (i.e., MMN is elicited) but it is initially "suppressed" in amplitude until later in the block when the evidence sufficiently counters the first impression. The authors suppose that the first-impression formed in the stable sequence may include a superordinate variable (or hyperprior, Bernado \& Smith, 2000) specifying the block length. The block-length model would be violated in the subsequent unstable sequence when the first blocktype stops too early. As a consequence, the first-impression about tones may drop to a lowconfidence weighting because the super-ordinate assumption was wrong. This could explain why MMN to the first-deviant becomes smaller at block onsets that reactivate a role assumption with low-confidence but increases over the block when counter-evidence accumulates with stability. This explanation would, in turn, imply that unstable sequences encountered in isolation (i.e., not 
following a stable sequence) would produce the pattern of data observed in the stable sequence above because the first-impression would simply be linked to the onset structure of this sequence.

In study 2, we used a between-subjects design to explore the longevity of the order-effects on MMN. Specifically, we exposed participants to four occurrences of either the stable sequences or unstable sequences to determine whether the patterns observed in study 1 survive repeated experience with the sequences. Repeated experience of a given sequence structure could eventually overpower any biases or assumptions that do not hold value in predicting the environment over the longer term (see Mathys, Daunizeau, Friston, \& Stephan, 2011). Study 2 also facilitated a test of the hypothesis that the unstable sequence data produced in study 1 were due the influence of the prior stable sequence structure and an additional test of whether adaptation at the end of the stable sequences could explain the unstable sequence data. If such adaptation effects were present, repeats of the stable sequences should be subject to the same influence in which the adaptation to the standard at the end of the sequence should lead to smaller MMN to this sound as a deviant in the subsequent repeat presentation.

\section{Method - Study 2}

\section{Participants.}

Participants included 30 (21 females; $18-27$ years, mean $=21$ years $\mathrm{SD}=2$ years $)$ naive healthy community volunteers and undergraduate students from the University of Newcastle, Australia meeting the same inclusion criteria as study 1 . Remuneration was offered as course credit to students and cash reimbursement to community volunteers. Written informed consent was obtained from all participants consistent with standards approved by the local Human Research Ethics Committee.

\section{Stimuli and sequences.}

The protocol was a modification of Todd et al.'s (2013a; described in study 1) multipletimescale paradigm comprising of four occurrences of either the stable sequence (stable condition) 
or the unstable sequence (unstable condition). For both conditions, a 1 min break occurred between each sequence repeat.

\section{Procedure}

Screening and EEG data acquisition procedure was identical to study 1. Condition allocation alternated with recruitment order (three males in the stable condition and six in the unstable; and mean age of 22 years in both subgroups). Sixteen participants were allocated to the stable condition and the remaining 14 participants to the unstable condition. Sequences were presented over headphones (Sennheiser HD280pro) while the participant viewed a film (sound muted with subtitles and instructed to focus on the film as per study 1).

\section{Data Analysis.}

EEG data analysis was identical to study 1 and generated ERPs for the period equating to the first- versus second-half of blocks for each tone, in each sequence and repeat. This resulted in 8 standard and 8 deviant ERP averages per tone (therefore 16 standard and 16 deviant per participant, per presentation). The minimum sweeps contributing to an average for any participant was 44 with the mean between 58 and 59 for all deviant waveforms. Eight difference waveforms were generated for each tone (first and second halves for each for the four sequences).

The mean-peak amplitudes was quantified as the maximum negativity in difference waveforms over a $20 \mathrm{~ms}$ period centered on the most negative point $100-250 \mathrm{~ms}$ following stimulus onset. The purpose was to determine whether the expected tone $\mathrm{x}$ half interactions seen in study 1 diminished with repeated presentations. Amplitudes were therefore compared in a mixed model ANOVA at F4 with condition (stable, unstable) as a between-groups factor and within-subjects factors of half $\left(1^{\text {st }}, 2^{\text {nd }}\right)$, deviant (first-deviant, second-deviant) and presentation (Sequence 1 , Repeat 1, Repeat 2, and Repeat 3). Paired t-tests were performed for planned comparisons using simple effects with p-value set at .05 . Effects on deviant versus standard tones are presented in supplementary data and confirm that the bias is present in response to deviants only.

\section{Results.}


Difference waveforms for first and second-deviant, across each block half, and each presentation, are presented for stable versus unstable sequences in Figure 4. Analysis revealed main effects of presentation $(F(3,84)=9.24, p<.001 \eta 2=.25)$, deviant $(F(1,28)=18.15, p<.001 \eta 2=$ $.39), \operatorname{half}(F(1,28)=17.80, p<.001 \eta 2=.38)$ and condition $(F(1,28)=27.64, p<.001 \eta 2=.50)$. While the main effect of condition is due to MMNs being larger overall in the stable group, the main effects of deviant, half and presentation were further modified by significant interactions between deviant and half $(F(1,28)=6.42, p<.05 \eta 2=.19)$ and deviant and presentation $(F(1,28)$ $=2.83, p<.05 \eta 2=.09$ ). From Figure 4 it is apparent that the deviant by half interaction occurs due to MMN being smaller in the first-half than the second-half of sequence blocks for the seconddeviant but not the first. This was confirmed in analyses conducted separately for the first and second deviant. For the first deviant there was a significant main effect of condition (MMN smaller in the unstable group, $F(1,28)=18.81, p<.001 \eta 2=.40)$ and presentation $(F(3,84)=5.79, p<$ $.001 \eta 2=.17)$, with the latter modified by a significant presentation $x$ half interaction $(F(3,45)=$ $6.30, p<.05 \eta 2=.11)$. This interaction was due to presentation affecting MMN size in the secondhalf of blocks (MMN decreasing over presentations, $F(3,84)=10.65, p<.001 \eta 2=.28)$ but not the first-half $(p=.76)$. MMN did not differ significantly over halves for the first-deviant in any of the four presentations ( $\mathrm{p}>.09$ for paired t-tests over halves in all stable and unstable sequences). In contrast, the analysis of second-deviant MMNs confirmed a main effect of half $(F(1,28)=33.12, p$ $<.001 \eta 2=.54)$ due to MMN incrementing across halves across all presentations. A main effect of presentation was also present $(F(3,26)=6.39, p<.001 \eta 2=.19)$ due to a reduction in MMN amplitude across repeating sequences. 


\section{A.First Deviant (60ms) B.Second Deviant (30ms)}

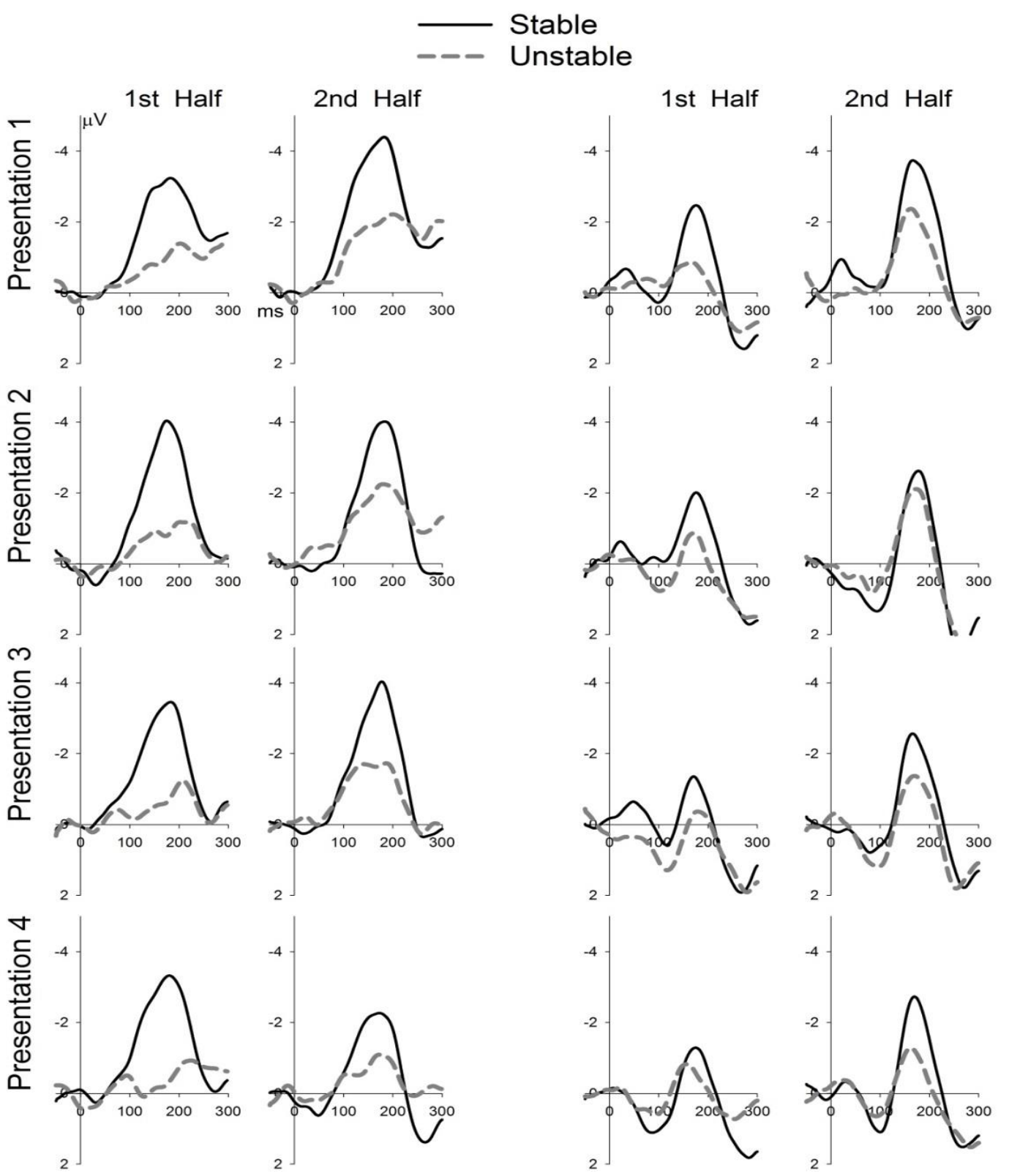

Figure 4. Difference waveforms for the first $(\mathrm{A}=60 \mathrm{~ms})$ and second $(\mathrm{B}=30 \mathrm{~ms})$ deviant in the $1^{\text {st }}$ and $2^{\text {nd }}$ half of stable (black solid lines) and unstable (grey broken lines) sequences. Difference waveforms are presented separately for data acquired in the first, second, third and fourth sequence presentation.

An additional analysis step was conducted on the stable sequence group data in light of the consistency of the differential effect of stability on the first and second-deviant MMNs over 
presentations. The data from the first and second halves of sequence blocks were further broken down into early and late blocks within the sequence. These were in turn averaged over sequences to create four MMNs per deviant type. Specifically, this produced an across-presentation average for the first-half and second-half of the early sequence block and the first-half and second-half of the later sequence block. This facilitated an examination of whether the stability patterns change from the first occurrence of the block to when the block occurred a second time. The average difference waves (minimum 90 sweeps) for each deviant and block are presented in Figure 5B and C along with a grand average of first-half and second-half stable sequence difference waves over the four presentations in Figure 5A. For transparency the grand average deviant and standard responses are also presented in the bottom panel of Figure 5.

It is clear from Figure 5B that although MMN to the first-deviant drops in amplitude from the early to the late block, the tendency for MMN to be more-or-less the same amplitude in both block halves remains constant. In a repeated measures ANOVA for first-deviant data with withinsubjects factors of period (early, late) and half (first, second) these visible patterns produce main effects of period only $(F(1,15)=23.04, p<.001, \eta 2=.61)$. In Figure $5 \mathrm{C}$ the MMN to the seconddeviant does not drop over early and late sequence blocks and appears to exhibit the same significantly smaller amplitude MMN for first-half of each block confirmed in a main effect of half only $(F(1,15)=17.18, p<.001, \eta 2=.53)$ in the corresponding repeated measures ANOVA. 


\section{A.STABLE SEQUENCE}
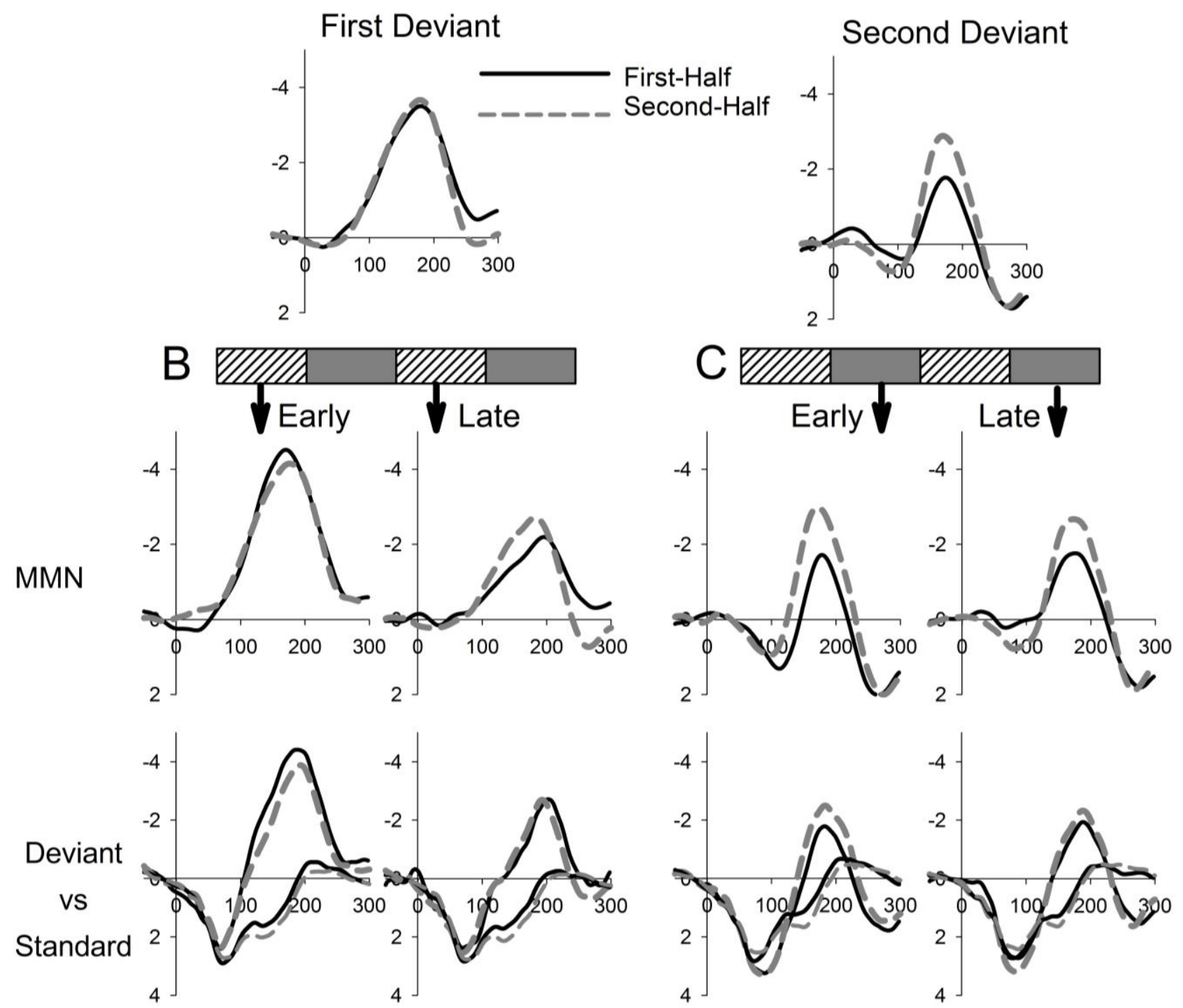

Figure 5. A. Difference waveforms for the first and second-deviant in the $1^{\text {st }}$ half (black solid lines) and $2^{\text {nd }}$ half of stable sequence blocks (grey broken lines) averaged over the four sequence presentations. B. Same as A but for the first-deviant tone only and with the response in early and late blocks separated. The top portion shows the difference waves and the bottom the deviant and standard ERPSs.C. Same as B but for the second-deviant tone only.

\section{Discussion.}

In study 2, we used a between-subjects design to examine how the order-driven bias in MMN amplitude was affected by repeated exposure to stable or unstable sequences across time. The data reveal a remarkably persistent pattern of differential effects on MMN amplitude for the 
first and second-deviant. The deviant by half interaction is consistent with the pattern seen for stable sequences in study 1 ; similar to study 1 , this difference is due to MMN amplitude being "suppressed" in the first-half of blocks for the second-deviant but not for the first, and this remains constant over sequence repeats. In contrast, the MMN to the first-deviant is not significantly smaller in the first-half of blocks. So the data across presentations are very consistent for the stable sequence and show that repeated exposure does not alter the different stability effects on MMN for the first and second-deviant tone.

The discussion for study 1 raised the possibility that the long period of repetition at the end of a stable sequence could cause a strong adaptation that might impact the response to tones in the following sequence. This was proposed as a possible explanation for why the pattern of stability effects reversed in the unstable sequence when it followed the stable sequence. Observations from study 2 provide compelling evidence that this is highly unlikely. If adaptation to standards in the final block of stable sequences impacted MMN to the first-deviant in the next sequence, this would act to "suppress" MMN amplitude to the first-deviant tones when the stable sequence is repeated. It is clear from Figures $4 \& 5$ that this is not the case; MMN to the first-deviant is always large (Figure 4) and is actually largest in the early blocks of the repeating stable sequences (Figure 5).

However, contrary to study 1 , the deviant by half interaction in unstable sequences in study 2 was not significantly different to that in stable sequences (i.e., the deviant by half interaction was not modified by condition). The repeats of the unstable sequence do not replicate the modulation patterns seen in study 1 (i.e., small MMN in the first-half of blocks for the first but not the seconddeviant). The effects on MMN amplitude in the unstable group in study 2 do not differ statistically from those in the stable repeat condition. It is also clear however, that the effects in the unstable group are much smaller and indeed, if analysed without the stable sequence group, produce a main effect of half only (second-half larger than first, see supplementary data). This observation is consistent with the proposition that the patterns in the unstable sequence in study 1 were due to the sequence being preceded by the stable sequence. When encountered in isolation (study 2), the 
unstable sequences produce minimal evidence of (or possible absence of) order-bias, which may reflect theory that any top-down influence on MMN amplitude will be less pronounced under conditions of low-confidence in the underlying model (Friston, 2005; Garrido et al., 2009; Lieder et al., 2013; Winkler, 2007). In the absence of any top-down bias, the results may be dominated by more local adaptation effects proposed by Costa-Faidella et al. (2011) where, with so many reversals, the adaptation effect on both tones as standards could begin to suppress the initial MMNs to the same tones as deviants leading to smaller MMNs in first-half data for both deviants.

\section{General Discussion \& Conclusions}

The studies presented in this paper demonstrate robust order-effects on modulation of MMN amplitude revealed in role-alternating sequence designs. If the system were realistically computing probabilities from tone occurrences, the patterns of change in MMN for both deviants should be equivalent. Instead, the evidence pertaining to the two tones and/or block types appears to be weighted differently. Although long time-scale adaptation could contribute to these order-effects (Costa-Faidella et al., 2011), it cannot account for why such effects can be altered by changing the behavioural relevance of the sounds (Mullens et al., 2014) and it does not appear to account for why the order-effects reverse if an unstable rapid role alternating sequence follows a stable slow role alternating sequence (study 1 and Todd et al., 2013).

It was hypothesised that repeated exposure to sequences (study 2) could result in diminishing bias as the auditory network learns over time that tones and block-types are equally probable. Instead, the pattern of bias remained. This suggests that the learning that generates the bias is stable or may even be reinforced with repeated exposure to sequences with the same initial structure (see Todd et al., 2013a for reversal of bias with reversal of deviant order).

We have elsewhere suggested (Todd et al., 2013a) that the "first-impression" bias we expand on in this paper is akin to the formation of an instantaneous prior with a lasting impact that is reactivated each time the roles reverse. MMN has generally been considered to reflect a very low 
level relevance filtering system, however this is challenged by the observation that these apparent instantaneous priors (biases) can have profound affects that are altered by previous experience with sound. As reviewed in the introduction, Mullens et al. (2014) showed that the appearance of the bias was a function of the behavioural relevance of sounds in a previous go-no-go task. The results suggest that the MMN prediction-error signal is capable of reflecting an integration of sound meaning or relevance with sound probability information (e.g. first-deviant is rare and potentially important; first standard is predicted and provides no new information). The observation that previous familiarity can affect MMN amplitude is also evident in studies showing larger MMN to recognised language (Jacobsen et al., 2004; Pulvermüller \& Shtyrov, 2006) and non-linguistic stimuli (Jacobsen, Schröger, Winkler, \& Horváth., 2005) than those with equivalent physical distinctiveness. Our observations indicate that these effects may not be solely related to familiarity but instead to what participants have to do in response to the sound (i.e., sound relevance). These propositions require additional investigation but invite the intriguing possibility that applications of MMN can be used to study how priors interact with evidence to influence predictive processes that filter relevance in the unattended auditory environment.

Although order biases have emerged in (and could be confined to) studies employing rolereversing paradigms, what the data clearly reveal is that we do not yet understand all of the factors that significantly impact MMN amplitude. It is therefore imperative that continued efforts are made to explore such phenomena, particularly those that challenge current assumptions about the conditions in which smaller MMN is observed. Certainly, current models of the inferential process appear incomplete because they would not predict the present data. Studies using the multitimescale paradigm have shown very long lasting cumulative effects of learning that appear to distort confidence in automatic perceptual inferences. A deeper understanding of how this learning impacts MMN amplitude in healthy populations will ultimately advance our understanding of the many ways in which the system underlying sensory predictions and MMN amplitude can be impaired in a variety of clinical groups including those with schizophrenia. Furthermore, paradigms 
that can dissociate adaptation effects on ERPs from those that might be attributed to active prediction-models can contribute to identifying the contribution of each of these elements to smaller MMN in clinical populations.

\section{Acknowledgements}

IW was supported by the Hungarian Academy of Sciences Lendulet grant (LP2012-36). JDF acknowledges receipt of an Australian Postgraduate Award scholarship. We thank Gavin Cooper for his assistance in programming these experiments. This research was supported by funds provided by the National Health and Medical Research Council of Australia (APP1002995). 


\section{Reference}

Atkinson, R. J., Michie, P. T., \& Schall, U. (2012). Duration mismatch negativity and P3a in firstepisode psychosis and individuals at ultra-high risk of psychosis. Biological psychiatry, 71, 98-104. doi: http://dx.doi.org/10.1016/j.biopsych.2011.08.023

Baldeweg, T. (2006). Repetition effects to sounds: evidence for predictive coding in the auditory system. Trends in Cognitive Sciences, 10, 93-94. doi:10.1016/j.tics.2006.01.010

Baldeweg, T., Klugman, A., Gruzelier J. H., \& Hirsch, S. R. (2002). Impairment in frontal but not temporal components of mismatch negativity in schizophrenia. International Journal of Psychophysiology, 43, 111-122.

Baldeweg, T., Klugman, A., Gruzelier, J., \& Hirsch, S. R. (2004). Mismatch negativity potentials and cognitive impairment in schizophrenia. Schizophrenia research, 69, 203-217.

Bendixen, A., Prinz, W., Horváth, J., Trujillo-Barreto, N. J., \& Schröger, E. (2008). Rapid extraction of auditory feature contingencies. Neuroimage, 41, 1111-1119. doi:10.1016/j.neuroimage.2008.03.040

Bernardo, J., \& Smith, A. (2000). Bayesian theory. New York, N.Y.: Wiley.

Bodatsch, M., Ruhrmann, S., Wagner, M., Müller, R., Schultze-Lutter, F., Frommann, I., ... \& Brockhaus-Dumke, A. (2011). Prediction of psychosis by mismatch negativity. Biological psychiatry, 69, 959-966. doi: http://dx.doi.org/10.1016/j.biopsych.2010.09.057 
Costa-Faidella, J., Grimm, S., Slabu, L., Díaz-Santaella, F., \& Escera, C. (2011). Multiple time scales of adaptation in the auditory system as revealed by human evoked potentials. Psychophysiology, 48, 774-783. doi: 10.1111/j.1469-8986.2010.01144.x.

Cowan, N., Winkler, I., Teder, W., \& Näätänen, R. (1993). Memory prerequisites of mismatch negativity in the auditory event-related potential (ERP). Journal of Experimental Psychology: Learning, Memory, and Cognition, 19, 909.

Dima, D., Frangou, S., Burge, L., Braeutigam, S., \& James, A.C. (2012). Abnormal intrinsic and extrinsic connectivity within the magnetic mismatch negativity brain network in schizophrenia: A preliminary study. Schizophr Res, 135, 23-27.

Frith, C. D. (2014). The cognitive neuropsychology of schizophrenia. Psychology Press.

Garrido, M. I., Friston, K. J., Kiebel, S. J., Stephan, K. E., Baldeweg, T., \& Kilner, J. M. (2008). The functional anatomy of the MMN: a DCM study of the roving paradigm. Neuroimage, $42,936-944$.

Gil-da-Costa, R., Stoner, G. R., Fung, R., \& Albright, T. D. (2013). Nonhuman primate model of schizophrenia using a noninvasive EEG method. Proceedings of the National Academy of Sciences, 110, 15425-15430. doi:10.1073/pnas.1312264110

Haenschel, C., Vernon, D. J., Dwivedi, P., Gruzelier, J. H., \& Baldeweg, T. (2005). Event-related brain potential correlates of human auditory sensory memory-trace formation. The journal of Neuroscience, 25, 10494-10501.

Jacobsen, T., Horváth, J., Schröger, E., Lattner, S., Widmann, A., \& Winkler, I. (2004). Preattentive auditory processing of lexicality. Brain and Language, 88, 54-67.

Jacobsen, T., Schröger, E., Winkler, I., \& Horváth, J. (2005). Familiarity affects the processing of task-irrelevant auditory deviance. Journal of Cognitive Neuroscience, 17(11), 170

Javitt, D. C., Grochowski, S., Shelley, A. M., \& Ritter, W. (1998). Impaired mismatch negativity $(\mathrm{MMN})$ generation in schizophrenia as a function of stimulus deviance, probability, and interstimulus/interdeviant interval. Electroencephalography and Clinical 
Neurophysiology/Evoked Potentials Section, 108, 143-153. doi:10.1016/S0168-

\section{$\underline{5597(97) 00073-7}$}

Javitt, D. C., Zukin, S. R., Heresco-Levy, U., \& Umbricht, D. (2012). Has an angel shown the way? Etiological and therapeutic implications of the PCP/NMDA model of schizophrenia. Schizophrenia bulletin, 38, 958-966. doi:10.1093/schbul/sbs069

Kujala, T., Tervaniemi, M., \& Schröger, E. (2007). The mismatch negativity in cognitive and clinical neuroscience: theoretical and methodological considerations. Biological psychology, 74(1), 1-19. doi:10.1016/j.biopsycho.2006.06.001

Lieder, F., Stephan, K. E., Daunizeau, J., Garrido, M. I., \& Friston, K. J. (2013). A neurocomputational model of the mismatch negativity. PLoS computational biology, 9, e1003288. doi:10.1371/journal.pcbi.1003288

Light, G. A., Swerdlow, N. R., Rissling, A. J., Radant, A., Sugar, C. A., Sprock, J., ... \& Braff, D. L. (2012). Characterization of neurophysiologic and neurocognitive biomarkers for use in genomic and clinical outcome studies of schizophrenia. PLoS One, 7(7), e39434.

Mathys, C., Daunizeau, J., Friston, K. J., \& Stephan, K. E. (2011). A Bayesian foundation for individual learning under uncertainty. Frontiers in human neuroscience, 5, 39. doi:10.3389/fnhum.2011.00039.

Mullens, D., Woodley, J., Whitson, L., Provost, A., Heathcote, A., Winkler, I., \& Todd, J. (2014). Altering the primacy bias-How does a prior task affect mismatch negativity? Psychophysiology, 51, 437-445. doi:10.1111/psyp.12190

Näätänen, R. (1992) In Attention and Brain Function. Hillsdale, NJ: Lawrence Erlbaum Associates (1992).

Näätänen R., Jacobsen, T., \& Winkler, I. (2005). Memory-based or afferent processes in mismatch negativity (MMN): a review of the evidence. Psychophysiology, 42, 25-32, 2005.

Näätänen, R., Kujala, T., \& Winkler, I. (2011). Auditory processing that leads to conscious perception: a unique window to central auditory processing opened by the mismatch 
negativity and related responses. Psychophysiology, 48, 4-22. doi:10.1111/j.1469$\underline{8986.2010 .01114 . x}$

Pulvermüller, F., \& Shtyrov, Y. (2006). Language outside the focus of attention: the mismatch negativity as a tool for studying higher cognitive processes. Progress in neurobiology, 79, 49-71. doi:10.1016/j.pneurobio.2006.04.004

Sadia, G., Ritter, W., \& Sussman, E. (2013). Category effects: Is top-down control alone sufficient to elicit the mismatch negativity (MMN) component?. Biological psychology, 92, 191-198.

Sams, M., Alho, K., \& Näätänen, R. (1983). Sequential effects on the ERP in discriminating two stimuli. Biological psychology, 17, 41-58. doi:10.1016/0301-0511(83)90065-0

Sato, Y., Yabe, H., Todd, J., Michie, P., Shinozaki, N., Sutoh, T., ... \& Kaneko, S. (2003). Impairment in activation of a frontal attention-switch mechanism in schizophrenic patients. Biological psychology, 62, 49-63. doi:10.1016/S0301-0511(02)00113-8

Semlitsch, H. V., Anderer, P., Schuster, P., \& Presslich, O. (1986). A solution for reliable and valid reduction of ocular artifacts, applied to the P300 ERP. Psychophysiology, 2, 695-703. http://dx.doi.org/10.1111/j.1469-8986.1986.tb00696.x

Shelley, A. M., Silipo, G., \& Javitt, D. C. (1999). Diminished responsiveness of ERPs in schizophrenic subjects to changes in auditory stimulation parameters: implications for theories of cortical dysfunction. Schizophrenia research, 37, 65-79. doi:10.1016/S0920$\underline{9964(98) 00138-8}$

Stephan, K. E., Baldeweg, T., \& Friston, K. J. (2006). Synaptic plasticity and dysconnection in schizophrenia. Biological psychiatry, 59, 929-939. doi:10.1016/j.biopsych.2005.10.005

Sussman, E. S. (2007). A new view on the MMN and attention debate: the role of context in processing auditory events. Journal of Psychophysiology, 21, 164-175.

Todd, J., Harms, L., Schall, U., \& Michie, P. T. (2013). Mismatch negativity: translating the potential. Frontiers in psychiatry, 4, doi: 10.3389/fpsyt.2013.00171 
Todd, J., Heathcote, A., Mullens, D., Whitson, L. R., Provost, A., \& Winkler, I. (2013a). What controls gain in gain control? Mismatch negativity (MMN), priors and system biases. Brain topography, 1-12. doi:10.1007/s10548-013-0344-4

Todd, J., Heathcote, A., Whitson, L. R., Mullens, D., Provost, A., \& Winkler, I. (2014). Mismatch negativity (MMN) to pitch change is susceptible to order-dependent bias. Frontiers in Neuroscience. doi:10.3389/fnins.2014.00180

Todd, J., Michie, P. T., Schall, U., Ward, P. B., \& Catts, S. V. (2012). Mismatch negativity (MMN) reduction in schizophrenia-Impaired prediction-error generation, estimation or salience? International Journal of Psychophysiology, 83, 222-231. doi:10.1016/j.ijpsycho.2011.10.003

Todd, J., Provost, A., Cooper, G. (2011). Lasting first impressions: A conservative bias in automatic filters of the acoustic environment. Neuropsychologia, 49, 3399-3405. doi:10.1016/j.neuropsychologia.2011.08.016

Todd, J., Provost, A., Whitson, L.R., Cooper, G., Heathcote, A. (2013b). Not so primitive: Context sensitive meta-learning about unattended sound sequences. Journal of Neurophysiology, 109, 99-105. doi:10.1152/jn.00581.2012

Ulanovsky, N., Las, L., Farkas, D., \& Nelken, I. (2004). Multiple time scales of adaptation in auditory cortex neurons. The Journal of Neuroscience, 24, 10440-10453.

Wacongne, C., Changeux, J. P., \& Dehaene, S. (2012). A neuronal model of predictive coding accounting for the mismatch negativity. The Journal of neuroscience, 32, 3665-3678. doi: $\underline{0.1523 / J N E U R O S C I .5003-11.2012>~}$

Widmann, A. \& Schröger E. (1999). Bootstrapping the distribution of the city-block distance between two repeated measures. Retrieved from University of Leipzig online website: http://www.uni-leipzig.de/ biopsych/widmann/minkowski.html

Winkler, I., \& Czigler, I. (2012). Evidence from auditory and visual event-related potential (ERP) studies of deviance detection (MMN and $\mathrm{vMMN}$ ) linking predictive coding theories and 
perceptual object representations. International Journal of Psychophysiology, 83(2), 132143. doi:10.1016/j.ijpsycho.2011.10.001

Winkler, I., Karmos, G., \& Näätänen, R. (1996). Adaptive modeling of the unattended acoustic environment reflected in the mismatch negativity event-related potential. Brain research, 742, 239-252. doi:10.1016/S0006-8993(96)01008-6

Winkler, I. (2007). Interpreting the mismatch negativity. Journal of Psychophysiology, 21, 147-163. doi: http://dx.doi.org/10.1027/0269-8803.21.34.147

Winkler, I., \& Schröger, E. (2015). Auditory perceptual objects as generative models: Setting the stage for communication by sound. Brain and language, 148, 1-22.

doi:10.1016/j.band1.2015.05.003 